\title{
Performance Characterization of PON Technologies
}

\author{
*Alex Vukovic, Khaled Maamoun, Heng Hua, Michel Savoie \\ Broadband Applications and Optical Networks, Communications Research Centre (CRC), Ottawa, \\ ON, Canada, K2H $8 \mathrm{~S} 2$
}

\begin{abstract}
The simulation models for a typical PON layout are developed and three major PON technologies are considered. The models support the analysis of various important characteristic parameters, namely: 1) link budget for acceptable losses from splices, attenuation and splitters, 2) link performance characterization based on data (BER, SNR) or video signal quality, and 3) linear and nonlinear fiber effects such as dispersion, PMD, self- and cross-modulation, FWM, etc. Analysis outcomes may be used to optimize the performance of the applied system design including fiber maximum length and type, the need to change some of the optical components (e.g. couplers, splitters, etc.) and digital links bit rate (e.g. $1.2 \mathrm{~Gb} / \mathrm{s}$ or $2.4 \mathrm{~Gb} / \mathrm{s}$ ) according to the required BER. The simulation models developed enable us with these detailed analyses of PON technologies without the need to build prototypes.
\end{abstract}

Keywords: Passive Optical Network, Bit Error Rate, Signal to Noise Ratio, Polarization Mode Dispersion, Four-Wave Mixing.

\section{INTRODUCTION}

Due to residential customer requests for high-speed communication, video, high definition multimedia capabilities, online interaction and peer-to-peer file transfer, the development and need for optical access technologies are growing. Fiber access network technologies are well suited to meet these requests. A network architecture based on Passive Optical Network PON technologies is elaborated along with options for different multiple access techniques.

A PON is a point-to-multipoint optical network. It consists of an Optical Line Terminator OLT located at the Central Office CO and a group of Optical Network Units ONUs at remote nodes located at the customer's premise. The connection between the OLT and ONUs is realized by a single fiber and the use of one or more optical splitters.

The network between the OLT and the ONUs is passive, meaning that it doesn't require any power supply. The presence of only passive elements in the network makes it relatively more fault tolerant, and decreases its operational and maintenance costs once the infrastructure has been laid down. A typical PON, as illustrated in Figure 1, uses a single wavelength for all downstream transmissions (from OLT to ONUs), and another wavelength for all upstream transmissions (from ONUs to OLT), multiplexed on a single fiber through coarse wavelength-division multiplexing CWDM [1].

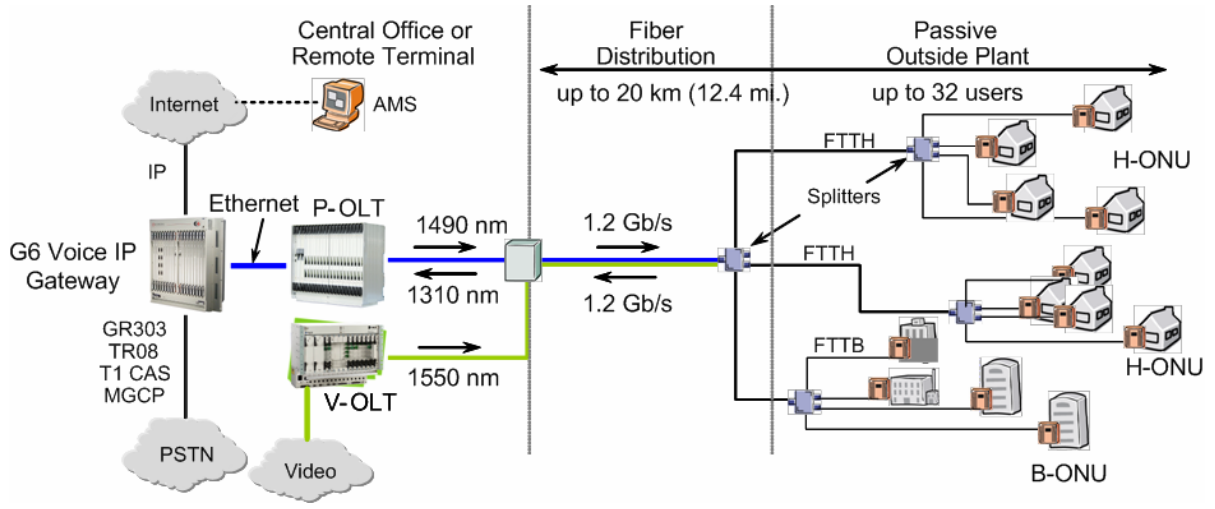

Fig. 1. Typical PON Architecture

*alex. vukovic @ crc .ca; phone 1613 998-2452; fax 1613 990-8382. 


\section{BACKGROUND AND CURRENT DEVELOPMENT}

Four major PON technologies are considered, namely Broadband PON (BPON), Ethernet PON EPON, Gigabit PON GPON, and Wavelength Division Multiplexing PON WDM PON. Table 1 summarizes the main differences between these four technologies whereas Table 2 lists the three PON classes as per ITU-T recommendation G.983.1. Each of them will be briefly described.

Table 1. PON Technologies

\begin{tabular}{|c|c|c|c|c|c|}
\hline & $\mathrm{A} / \mathrm{BPON}$ & $\begin{array}{c}\text { EPON } \\
(\text { GEPON) }\end{array}$ & GPON & 10 GEPON & WDM PON \\
\hline Standard & ITU G.983 & IEEE802ah & ITU G.984 & IEEE P802.3av & ITU G.983 \\
\hline Data Packet Cell Size & 53 bytes & 1518 bytes & 53 to 1518 bytes & 1518 bytes & Independent \\
\hline Maximum Downstream Line Rate & $622 \mathrm{Mbps}$ & 1.2 Gbps & 2.4 Gbps & $\begin{array}{c}\text { IP; } 2.4 \text { Gbps, } \\
\text { Broadcast; } 5 \text { Gbps } \\
\text { On-demand; } 2.5 \\
\text { Gbps }\end{array}$ & 1-10 Gbit/s per channel \\
\hline Maximum Upstream Line Rate & 155/622 Mbps & 1.2 Gbps & 1.2 Gbps & 2.5 Gbps & 1-10 Gbit/s per channel \\
\hline Downstream wavelength & $\begin{array}{l}1490 \text { and } 1550 \\
\text { nm }\end{array}$ & $1550 \mathrm{~nm}$ & 1490 and $1550 \mathrm{~nm}$ & $1550 \mathrm{~nm}$ & $\begin{array}{c}\text { Individual } \\
\text { wavelength/channel }\end{array}$ \\
\hline Upstream wavelength & $1310 \mathrm{~nm}$ & $1310 \mathrm{~nm}$ & $1310 \mathrm{~nm}$ & $1310 \mathrm{~nm}$ & $\begin{array}{c}\text { Individual } \\
\text { wavelength/channel }\end{array}$ \\
\hline Traffic Modes & ATM & Ethernet & $\begin{array}{c}\text { ATM Ethernet or } \\
\text { TDM }\end{array}$ & Ethernet & Protocol Independent \\
\hline Voice & ATM & VoIP & TDM & VoIP & Independent \\
\hline Video & $1550 \mathrm{~nm}$ overlay & $\begin{array}{c}1550 \mathrm{~nm} \\
\text { overlay/IP }\end{array}$ & $\begin{array}{l}1550 \mathrm{~nm} \text { overlay/ } \\
\text { IP }\end{array}$ & IP & 1550 nm overlay/ IP \\
\hline Max PON Splits & 32 & 32 & 64 & 128 & 16/100’s \\
\hline Max Distance & $20 \mathrm{Km}$ & $20 \mathrm{Km}$ & $60 \mathrm{Km}$ & $10 \mathrm{Km}$ & $20 \mathrm{Km}$ \\
\hline Average Bandwidth per User & $20 \mathrm{Mbit} / \mathrm{s}$ & $60 \mathrm{Mbit} / \mathrm{s}$ & $40 \mathrm{Mbit} / \mathrm{s}$ & $20 \mathrm{Mbit} / \mathrm{s}$ & $\begin{array}{l}\text { Up to } \\
10 \mathrm{Gbit} / \mathrm{s}\end{array}$ \\
\hline
\end{tabular}

Table 2. PON Classes

\begin{tabular}{|l|c|c|c|}
\hline & Class A (622 Mbps only) & Class B & Class C \\
\hline Minimum loss & $5 \mathrm{~dB}$ & $10 \mathrm{~dB}$ & $15 \mathrm{~dB}$ \\
\hline Maximum loss & $20 \mathrm{~dB}$ & $25 \mathrm{~dB}$ & $30 \mathrm{~dB}$ \\
\hline ONUs Max. No. & Up to 8 & Up to 16 & Up to 32 \\
\hline
\end{tabular}

\subsection{APON / BPON}

ATM Passive Optical Network APON was initiated in 1995 by ITU/FSAN and standardized as ITU-T G.983. In 1999, ITU adopted FSAN's APON standard. APON was the first PON based technology developed for FTTH deployment as most of the legacy network infrastructure was ATM based. There are different PON Technologies available today. Since the services offered by this architecture are not only the ATM based services but also video distribution, leased line services and Ethernet access and to express the broadband capability of PON systems APON was renamed as Broadband Passive Optical Network BPON. BPON was standardized by ITU recommendations G.983.1, .2, .3, and .4. BPON has two key advantages, first it provided a 3rd wavelength for video services, and second it is a stable standard that re-uses ATM infrastructures. ITU-T recommendation G.983.1 defines three classes of performance namely Class A, Class B and Class C. 


\subsection{GPON}

The progress in the technology, the need for larger bandwidths and the complexity of ATM forced the FSAN group to look for a better technology. Gigabit Passive Optical Network GPON standardization work was initiated by FSAN in the year 2001 for designing networks over 1Gbps. GPON architecture offers converged data and voice services at up to 2.5 Gbps. GPON enables transport of multiple services in their native formats, specifically TDM and data. In order to enable easy transition from BPON to GPON, many functions of BPON are reused for GPON. In January 2003, the GPON standards were ratified by ITU-T and are known as ITU-T Recommendations G.984.1, G.984.2 and G.984.3. GPON uses the Generic Framing Procedure GFP protocol to provide support for both voice and data oriented services. A big advantage of GPON over other schemes is that interfaces to all the main services are provided and in GFP enabled networks packets belonging to different protocols can be transmitted in their native formats.

\subsection{EPON}

Ethernet equipment vendors formed the Ethernet in the First Mile Alliance EFMA to work on an architecture for FTTH as Ethernet is a dominant protocol in Local Area Networks. EPON based FTTH was adopted by IEEE standard IEEE802.3ah in September 2004. Adopting Ethernet technology in the access network would result in a uniform protocol at the customer end simplifying network management. A single protocol for the Local Area Network, Access Network and Backbone network enables easy rollout of FTTH. EPON standards networking community renamed the term 'last mile' to 'first mile' to symbolize the importance and significance of the access part of the network. EFM introduced the concept of Ethernet Passive Optical Networks EPONs, in which a point to multipoint P2MP network topology is implemented with passive optical splitters. EPON, is largely a vendor-driven standard and it is fundamentally similar to ATM-PON but transports Ethernet frames/packets instead of ATM cells. It specified minimum standardization and product differentiation, also it has decided not to standardize the Bandwidth allocation algorithm DBA, TDM and ATM support, Security, Authentication, WDM Overlay Plan, support for Analog Video Protection, Diagnostics, Monitoring, Compliance with existing OSS leaving these to the vendors to choose the best.

\subsection{WDM PON}

Wavelength Division Multiplexing Passive Optical Network WDM PON is the next generation in the development of access networks and offers highest bandwidth. Though it will be some time before there is affordable WDM PONs some vendors are introducing products that can put more wavelengths onto a PON. Vendors are of the opinion that a Coarse CWDM PON can support 3 to 5 wavelengths, while supporting more than 5 wavelengths requires a DWDM overlay. In a WDM PON architecture, ONUs operate on different wavelengths and hence higher transmission rates can be achieved. Much research was focused on enhancing WDM PON's ability to serve larger numbers of customers in an attempt to increase revenue from invested resources. As a result, some hybrid structures have been proposed where both WDMA and TDMA modes are used to increase the number of potential users. For DWDM, the ONUs require expensive, frequency-stable, temperature-controlled lasers. The OLT puts all the wavelengths onto the shared feeder fiber and the splitters replicate the wavelengths to each home [3].

\section{PON MODEL DEVELOPMENT}

We developed a simulation model based on the EPON architecture as it has symmetrical line rates for both upstream and downstream (as illustrated in Figure 1). For APON, BPON and GPON, slight modifications to the simulation model in terms of the bit rates and traffic directions need to be made as the infrastructure for all of these architectures is the same. While in WDM PON, we need to redesign the entire network to either Composite PON (CPON), LARNET (Local Access Router Network), or RITENET (Remote Interrogation of Terminal Network). These three models are the commonly deployed WDM PON architectures. Figure 2 demonstrates the EPON model considered in this paper. The simulation is performed for this model using RSoft's Optsim tool.

\section{SIMULATION DEVELOPMENT}

In the simulation, we considered each of the "triple play" services: voice, data, and video services. Data and voice can be treated as one simulation entity as VoIP is the technique used in EPON for voice traffic, the current alternative to traditional PSTN with POTS.

A CATV network uses multiple frequency blocks or channels to deliver programming. In North America, each channel is $6 \mathrm{MHz}$ wide. Current state-of-the-art systems transport frequencies from 50 to $870 \mathrm{MHz}$, delivering 133 channels. 


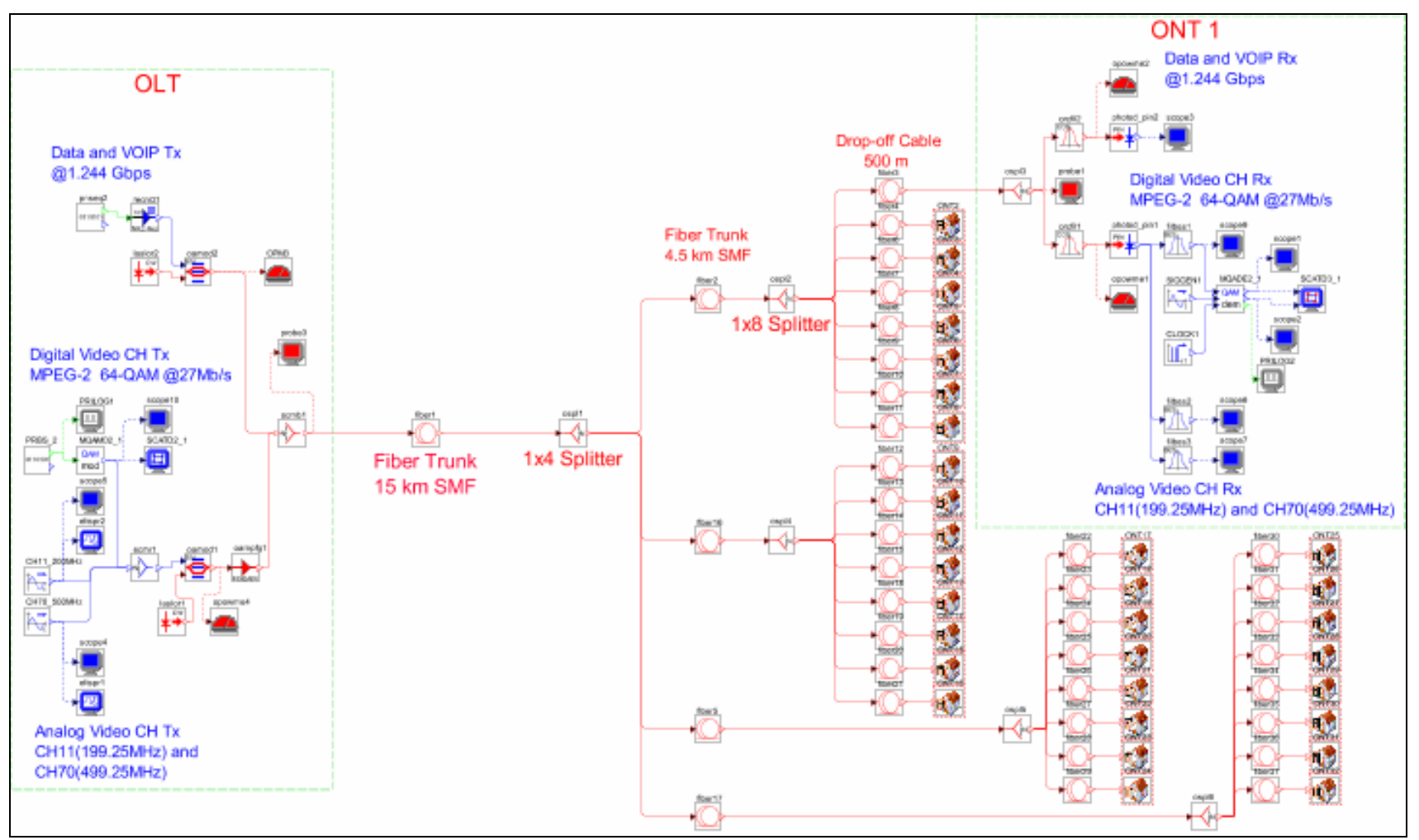

Fig. 2. Typical PON Architecture

A video channel may be in either analog (NTSC) or digital (64 quadrature amplitude modulation QAM or 256 QAM) format. In analog format, a single video program occupies one channel. In digital format, many MPEG-2 compressed video programs can be multiplexed into one channel. Depending on the modulation technique (64 QAM or 256 QAM) and video compression, more than 10 video programs can share the same channel. The primary advantage of the analog format is that it can be displayed on existing televisions without the need for a set-top box (STB) converter. Digital format, on the other hand, requires an STB for each television but has the advantages of being able to carry more programs and to easily encrypt programs to prevent unauthorized viewing. Perhaps an even greater advantage of the digital format is that it requires significantly less power than analog for the same picture quality. Channels using 256 QAM to carry digital video are usually transported at $-5 \mathrm{dBc}$ relative to analog channels [4]. A digital Video component is represented as 64-QAM CATV SCM signal, whereas an Analog Video component is represented by two analog (RF) channels: CH11 (199.25MHz) and CH70 (499.25MHz) only for simplicity.

The simulation model consists of three sections: 1) OLT, 2) ONU and 3) Distribution network. Table 3 lists optical interface parameters of $1244 \mathrm{Mbit} / \mathrm{s}$ downstream direction as specified is G.984.2.

Table 3. G.984.2 - Optical interface parameters of 1244 Mbit/s downstream direction

\begin{tabular}{|l|l|c|c|c|}
\cline { 2 - 5 } \multicolumn{1}{c|}{} & ODN Class & A & B & C \\
\hline \multirow{3}{*}{ OLT Transmitter (optical interface) } & Mean launched power MIN (dBm) & -4 & +1 & +5 \\
\cline { 2 - 5 } & Mean launched power MAX (dBm) & +1 & +6 & +9 \\
\cline { 2 - 5 } & Extinction ratio & \multicolumn{3}{|c|}{ More than 10} \\
\hline \multirow{3}{*}{ ONU Receiver (optical interface) } & Minimum sensitivity $(\mathrm{dBm})$ & -25 & -25 & -26 \\
\cline { 2 - 5 } & Minimum overload $(\mathrm{dBm})$ & -4 & -4 & -4 \\
\cline { 2 - 5 } & Bit error ratio & \multicolumn{3}{|c|}{ Less than $10^{-10}$} \\
\hline
\end{tabular}




\subsection{OLT}

Data/voice transmitter consist of 1.25 Gbps PRBS generator, NRZ driver, CW externally modulated laser at $1490 \mathrm{~nm}$ wavelength, and Mach-Zehnder external modulator (as shown in Figure 3).

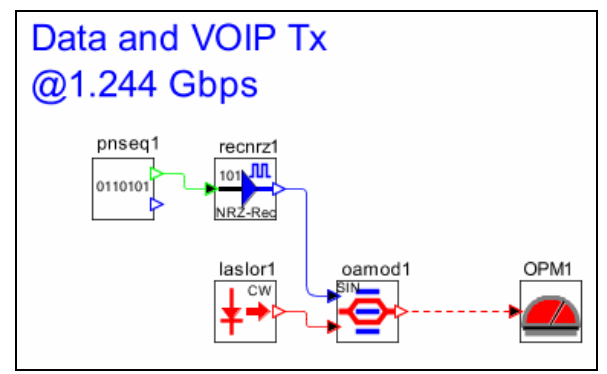

Fig. 3. Data/voice Transmitter

Video transmitter consists of PRBS generator and 64-QAM encoder for the digital part, two sine tone generators for the analog part, electrical combiner, CW externally modulated laser at 1550nm wavelength, and Mach-Zehnder external modulator. A 20dB EDFA booster is used at the output. The layout of the video transmitter section is illustrated in Figure 4).

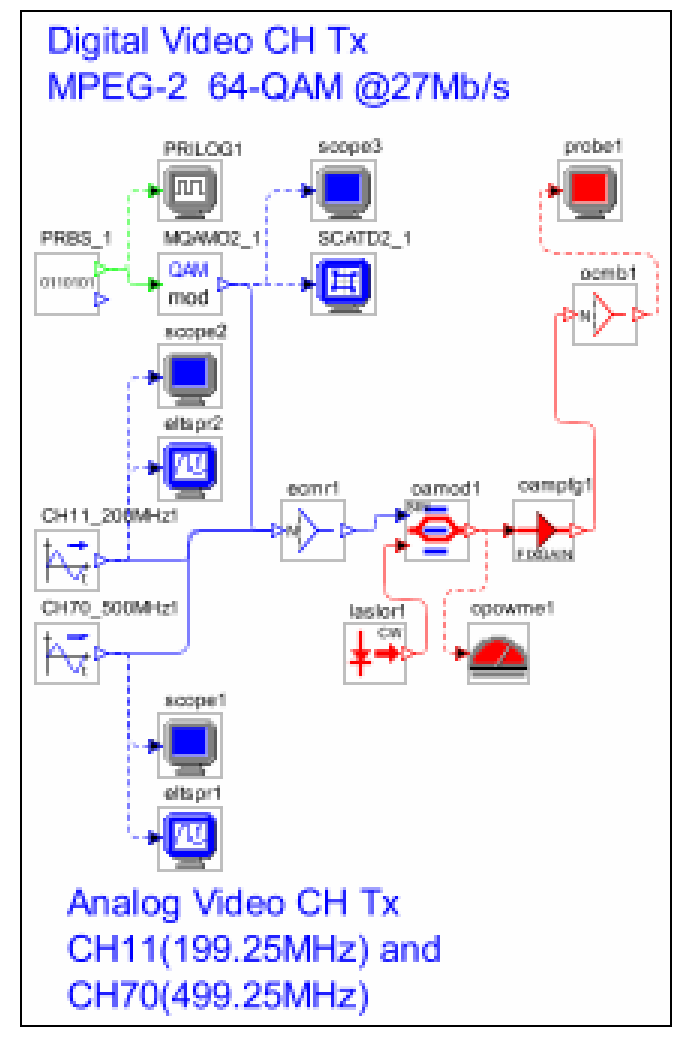

Fig. 4. CATV Transmitter

\subsection{Distribution Network}

Total fiber length is about $20 \mathrm{~km}$. Each of 32 (class C) paths goes through 3 splitters and 6 splices. Corning SMF28e, an ITU-T G.562 compliant fiber is simulated. All physical effects in the fiber are taken into account: attenuation, dispersion, PMD, four-wave mixing, self/cross-phase modulation, SBS, Raman crosstalk, etc. 


\subsection{ONU}

Triple-service ONU consists of Data/VoIP and video receivers. Data/Voice receiver consist of optical filter, and PIN (as shown in Figure 5).

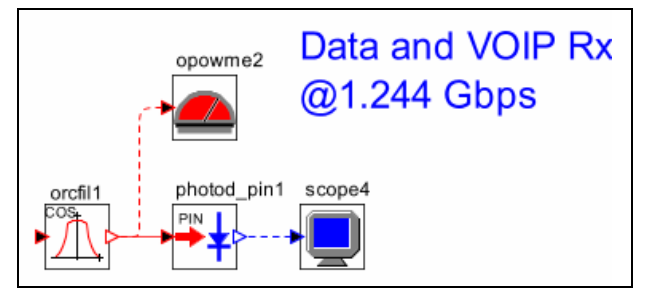

Fig. 5. Data/Voice Receiver

Video receiver consists of optical filter, PIN, electrical filters, sine wave generator, clock generator, QAM demodulator. For analysis purposes, we added measurement blocks in different places at both the transmitter and receiver section. Both analog and digital CATV receiver sections are illustrated in Figure 6.

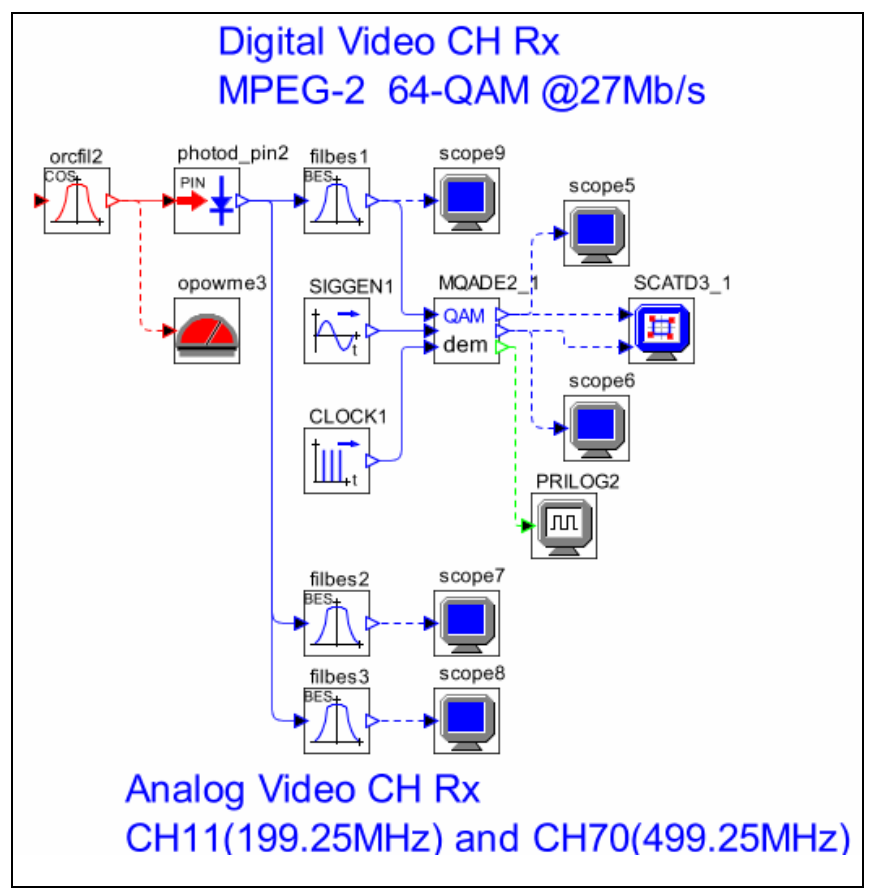

Fig. 6. CATV Receiver

\section{ANALYSIS OF RESULTS}

The performance measures for the Data/Voice analysis is the Eye-diagram and for CATV analysis are the multi-level Eye-diagram, the Carrier-to-Noise Ratio CNR, the Composite Second Order CSO, and the Composite Triple Beat CTB. Each simulation runs lasts $5000 \mathrm{~ns}$ in order to obtain sufficient data for the analyses and graphs.

\subsection{Distribution Network}

The optical power at the Transmitter section was $6.85 \mathrm{dBm}$ and the optical power at the Receiver section was -16.92 $\mathrm{dBm}$. The total signal attenuation from fiber spans and splitters is about $24 \mathrm{~dB}$. This value is in the range specified by ITU-T Recommendation G.984.2. (as illustrated in Table 2). 


\subsection{Data/Voice}

The Eye-diagram at the receiver is shown in Figure 7. In the ITU-T Rec. G.984.2, general transmitter pulse-shape characteristics including rise time, fall time, pulse overshoot, pulse undershoot, and ringing, all of which should be controlled to prevent excessive degradation of the receiver sensitivity, are specified in the form of a mask of the transmitter Eye-diagram. The standard specified that the mask of the Eye-diagram for the downstream direction signal is applied from the first bit of the preamble to the last bit of the signal inclusive. In our simulation, there are no violations spotted in the Eye diagram. The spectrum of the optical carrier and the electrical data pattern at the receiver side are illustrated in Figures 8 and 9.

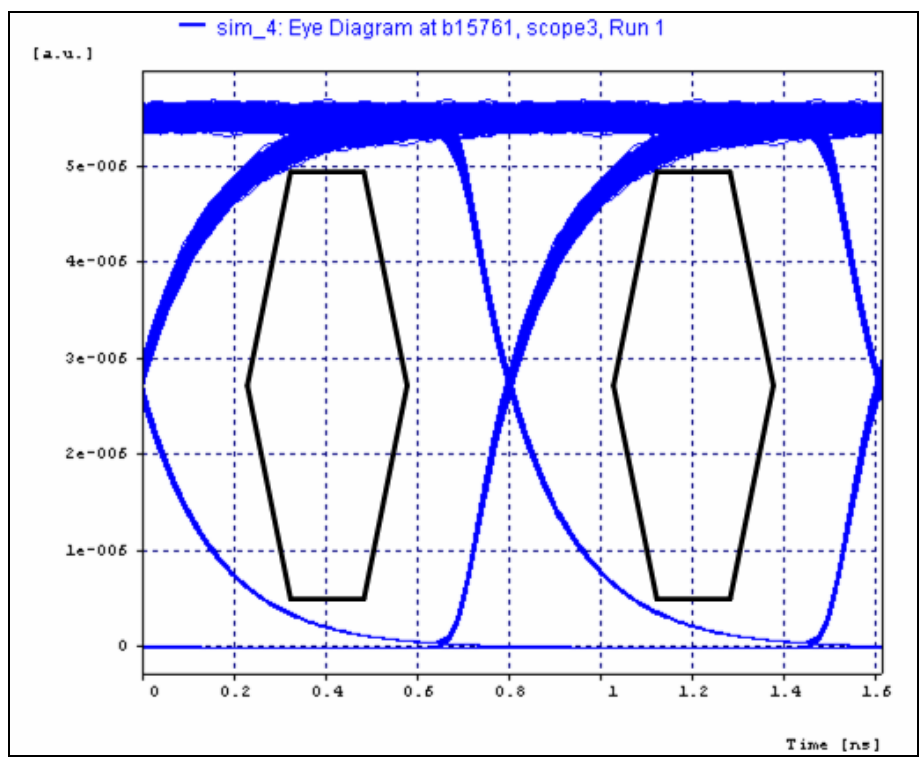

Fig. 7. Data/Voice Eye-diagram with ITU-T Rec. G.984.2 mask

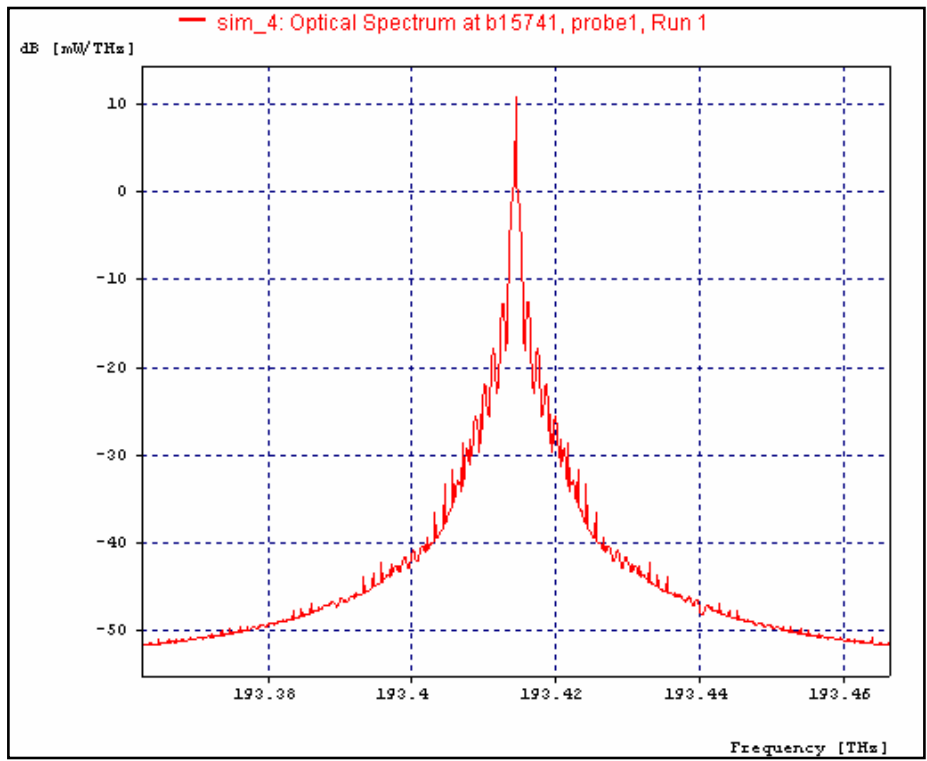

Fig. 8. Data/voice Optical Carrier (1490nm) Spectrum 


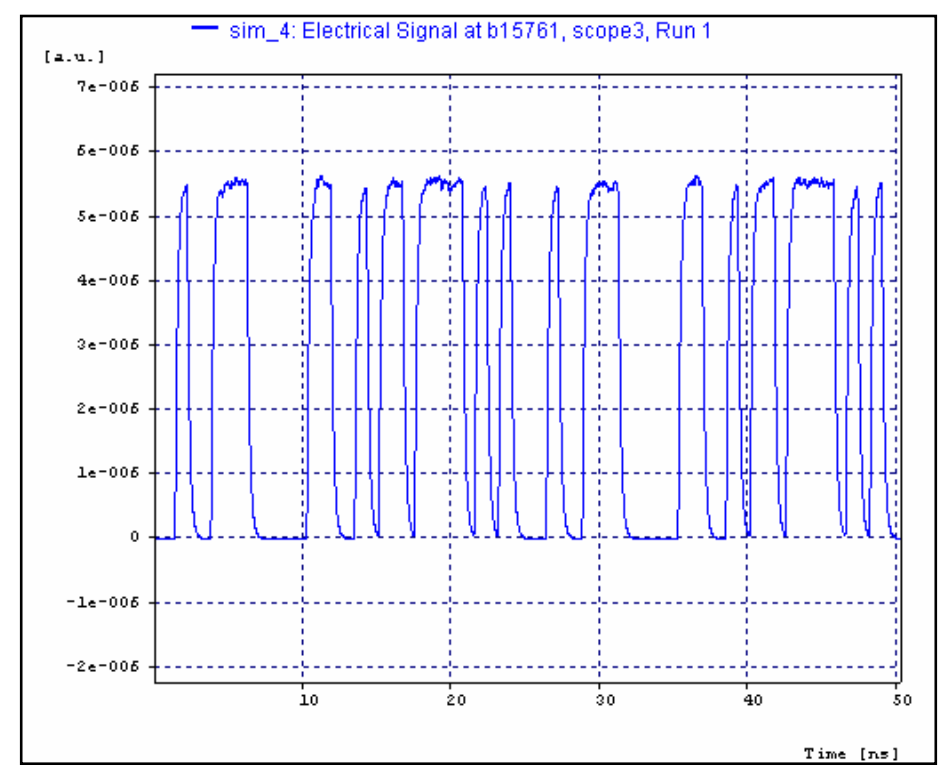

Fig. 9. Data/Voice Received Electrical Data Pattern

\subsection{CATV}

The electrical spectrum for the two pilot tones simulating CH11 and CH70 analog CATV at the receiver side is illustrated in Figure 10. The 6-level logical signal of the 64-QAM is presented in Figure 11 and the multi-level Eyediagram at the receiver is shown in Figure 12.

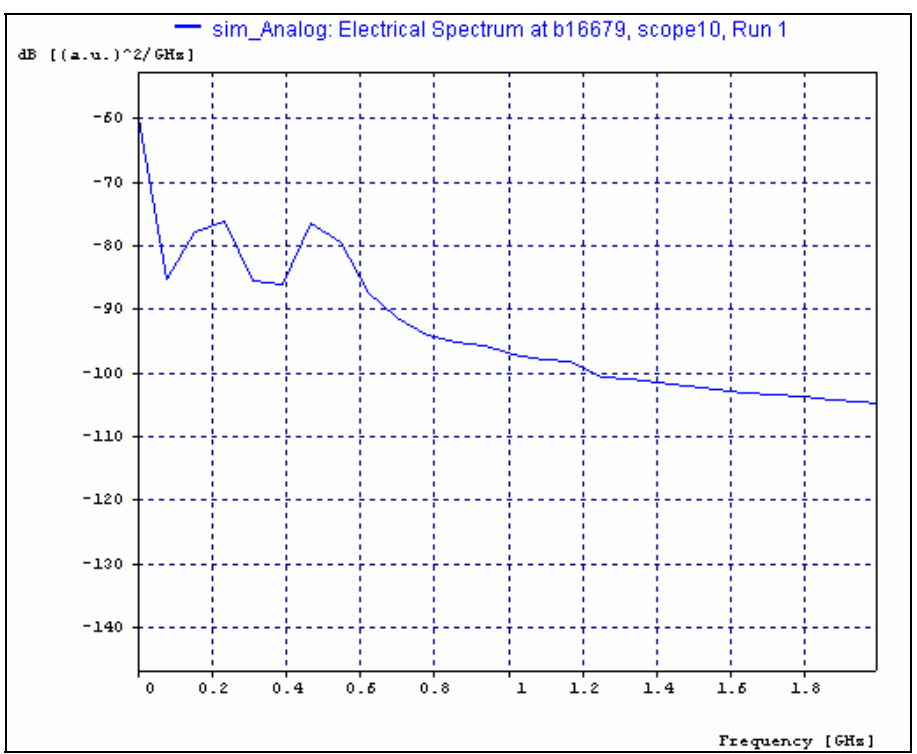

Fig. 10. Electrical Spectrum of the Simulated CH11 and CH70 Analog CATV 


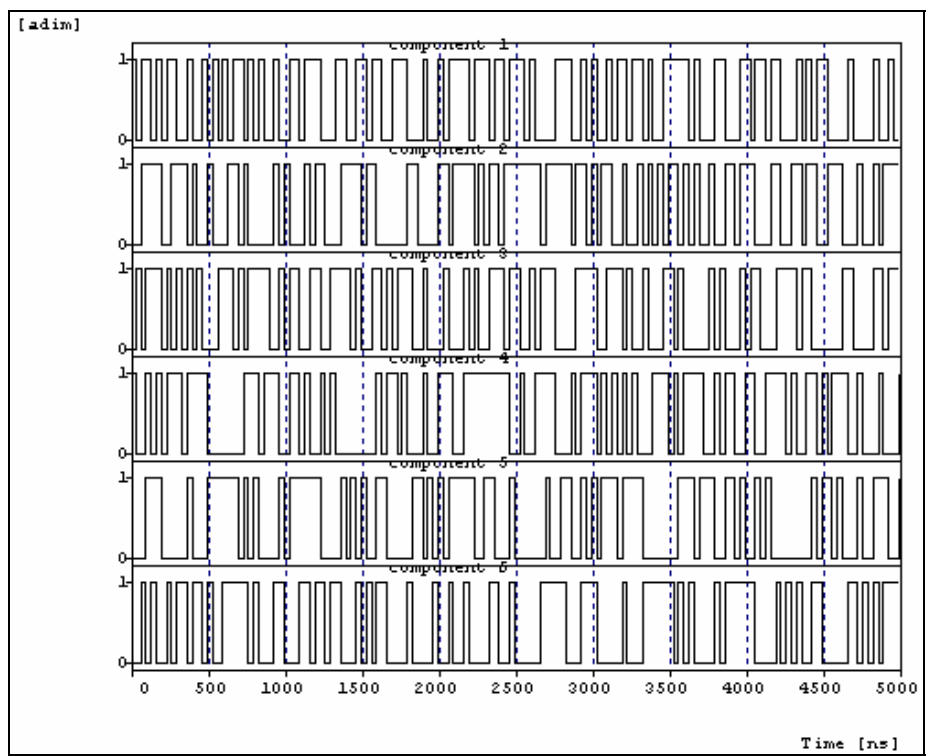

Fig. 11. Received 64-QAM Multi-level Logical Data Pattern

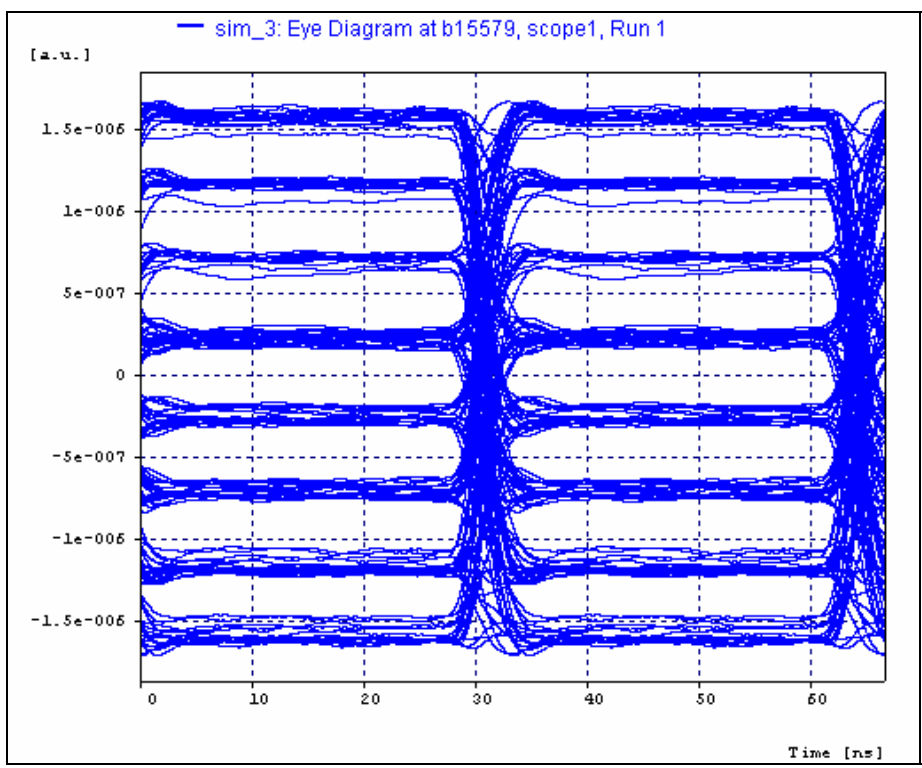

Fig. 12. Received 64-QAM Multi-level Eye-diagram

The 64-QAM MPEG-2 digital CATV signal constellation diagram at the transmitter and receiver are shown in Figures 13 and 14. The 64-QAM representation points in the diagram are connected according to its transmission sequence. The measured parameters are as follows: CNR was $54 \mathrm{~dB}$, CSO was $-53.2 \mathrm{dBc}$, and CTB was $-53.2 \mathrm{dBc}$. The Federal Communications Commission FCC has established minimum standards for video quality when providing analog video services [14]. The minimum standards are CNR greater than or equal to $43 \mathrm{~dB}$, CSO less than or equal to $-51 \mathrm{dBc}$, and CTB less than or equal to $-51 \mathrm{dBc}$. The Society of Cable Telecommunication Engineers SCTE has established a standard of not worse than $-53 \mathrm{dBc}$ for CSO and CTB, a level that produces video imperfections that many consider to be imperceptible [15]. While the FCC and SCTE requirement for CNR is $43 \mathrm{~dB}$, most CATV providers seek to deliver better video quality, with a CNR greater than or equal to $47 \mathrm{~dB}$. 


\section{CONCLUSION}

The intention of this paper is simply to develop a simulation model that can be used in PON technology design analyses. After PON link performance requirements were established, the proper components could be selected and their simulation results are used as a feedback to change the architecture, components, and/or these requirements. The design process thus becomes iterative. The impact of fiber nonlinearities and the use of different fiber types can be addressed in the simulation which makes the design process fairly simple. With the different PON technologies and configurations in terms of line rates, split ratios, and triple play broadband services, the use of simulation helps us to focus on identifying the right design and making decisions regarding how to deploy PONs to address the service needs without getting bogged down on a technology debate. In conclusion, FTTH networks using PON technologies can be characterized and maintained at every stages of the design layout using the simulation which reduces the requirement of expensive test and measurement equipment in complex testbeds.

\section{REFERENCES}

1. K. M. Sivalingam and S. Subramaniam, Emerging Optical Network Technology; Architecture, Protocols, and Performance, Springer Science+Business Media, Inc., New York, 2005.

2. Allied Telesyn, Inc., “Active vs. PON FTTx Technology Choices,” Technical Brief (A), 1-10 (2004).

3. J. S. Shaik and N R Patil, "FTTH Deployment Options for Telecom Operators," Sterlite Optical Technologies Ltd., White Paper, 1-9 (2005).

4. B. Perkins, “The Art Of Overlaying Video Services On A BPON," Bechtel Telecommunications Technical Journal 2(2), 61-69 (2004).

5. MAXIM High-Frequency/Fiber Communications Group, "HFAN-9.0.3: APON Class A, B, \& C Physical Layer Requirements," Application Note 1102(0), 1-4 (2002).

6. EXFO, "Fiber Optic Testing Challenges in Point-to-Multipoint PON Testing," Application Note 110, 1-10 (2005).

7. D. Gutierrez et al., "FTTH Standards, Deployments and Research Issues," Photonics and Networking Research Lab., Proceedings of JCIS '05, 1358-1361 (2005).

8. M. Abrams et al., "FTTP Deployments in the United States and Japan - Equipment Choices and Service Provider Imperatives", Journal of Lightwave Technology 23(1), 236-246 (2005).

9. Nortel Networks, "Unleashing the power of PON," White Paper, 1-16 (2004).

10. Ian Cooper et al., "Video over BPON with Integrated VDSL," FUJITSU Sci. Tech. J. 37(1), 87-96 (2001).

11. A. Banerjee et al., "Wavelength-division-multiplexed passive optical network (WDM-PON) technologies for broadband access: a review," Journal of Optical Networking 4(11), 737-758 (2005).

12. ITU-T Recommendation G.983.1, 2005.

13. ITU-T Recommendation G.984.2, 2003.

14. Code of Federal Regulations, Title 47 Part 76.605, October 1, 2003.

15. ANSI/SCTE 40 2004, Digital Cable Network Interface Standard, 2004.

\section{ACKNOWLEDGEMENT}

The research is jointly supported by the Natural Sciences and Engineering Research Council (NSERC) Canada under the research program "Dynamically Provisioned Wavelength Networks" and by the Broadband Applications and Optical Networking Group, Communications Research Centre (CRC), Ottawa, Canada.

We would like to thank RSoft for providing us with valuable consultation in developing simulation model. 